\title{
Characterization of a nondestructive beam profile monitor using luminescent emission
}

\author{
A. Variola, ${ }^{*}$ R. Jung, and G. Ferioli \\ AB Division, CERN, CH-1211 Geneva 23, Switzerland
}

(Received 7 June 2005; revised manuscript received 7 August 2007; published 12 December 2007)

\begin{abstract}
The LHC (large hadron collider) [LHC study group: LHC. The large hadron collider conceptual design; CERN/AC/95-05] is the future $p$ - $p$ collider under construction at CERN, Geneva. Over a circumference of $26.7 \mathrm{~km}$ a set of cryogenic dipoles and rf cavities will store and accelerate proton and ion beams up to energies of the order of $7 \mathrm{TeV}$. Injection in LHC will be performed by the CERN complex of accelerators, starting from the source and passing through the linac, the four booster rings, the proton synchrotron (PS), and super proton synchrotron (SPS) accelerators. One of the main constraints on LHC performance is emittance preservation along the whole chain of CERN accelerators. The accepted relative normalized emittance blowup after filamentation is $\pm 7 \%$. To monitor the beam and the emittance blowup process, a study of different prototypes of nonintercepting beam profile monitors has been performed. In this context a monitor using the luminescent emission of gases excited by ultrarelativistic protons $(450 \mathrm{GeV})$ was developed and tested in the SPS ring. The results of beam size measurements and their evolution as a function of the machine parameters are presented. The image quality and resolution attainable in the LHC case have been assessed. A first full characterization of the luminescence cross section, spectrum, decay time, and afterglow effect for an ultrarelativistic proton beam is provided. Some significant results are also provided for lead ion beams.
\end{abstract}

DOI: 10.1103/PhysRevSTAB.10.122801

PACS numbers: 41.85.Ew, 07.60.- j, 07.30.-t

\section{INTRODUCTION}

By luminescence, one usually means the deexcitation of an electronically excited atom or molecule with consequent radiative emission [1]. While electronic transitions can be considered as luminescence sources in atoms, more complex considerations should be made in a molecule owing to vibrational and rotational degrees of freedom. In this case, the increase in the number of possible excited states allows narrower energy transitions. For those in the order of some $\mathrm{eV}$, the corresponding emission is in the near $\mathrm{UV}$ and in the visible range. This makes it possible to work in a domain in which guiding and imaging components, like fast detectors, can be easily found on the market. An attempt to measure beam size and consequently the luminosity by means of nitrogen deexcitation at very low pressure $\left(10^{-9}-10^{-8}\right.$ Torr $)$ was first made at CERN in the ISR rings [2]. Later nitrogen was successfully used in LANL [3] with an $80 \mathrm{keV}$ proton beam for profile monitoring. The choice of nitrogen molecules $\left(\mathrm{N}_{2}\right)$ as a radiator appears appropriate especially owing to the features of the ionized $\left(\mathrm{N}_{2}{ }^{+}\right)$state: (i) high cross section in the near UV range $\left(\sigma \sim 330 \times 10^{-19} \mathrm{~cm}^{2}\right.$ at $391.4 \mathrm{~nm}$ for $200 \mathrm{keV}$ protons) [4]; (ii) emission in the near UV-visible range (2 main peaks at $391.4 \mathrm{~nm}$ and $427.3 \mathrm{~nm}$ ); (iii) compatibility with vacuum systems; (iv) relatively short radiation decay time of the order of $60 \mathrm{~ns}$ (at zero pressure) with a slope

\footnotetext{
*Corresponding author.

Presently at Laboratoire de l'Accélérateur Linéaire, Université Paris-Sud, Bâtiment 200, France. alessandro.variola@lal.in2p3.fr
}

depending slightly on pressure: $\sim 1.6 \times 10^{7} \mathrm{sec}^{-1} \mathrm{Torr}^{-1}$ [5].

According to the energy scaling given by the BetheBloch theory, the high-energy cross section should not provide enough photon flux for beam profile measurements at energies higher than a few $\mathrm{MeV}$ (in the case of proton beams) [6]. Figure 1 illustrates the scaling and indicates the expected energy loss for the super proton synchrotron (SPS) range. Furthermore, a dedicated cross-section experiment performed in the proton synchrotron (PS) [7] showed a reduced cross section in respect to the BetheBloch scaling of the $200 \mathrm{keV}$ results.

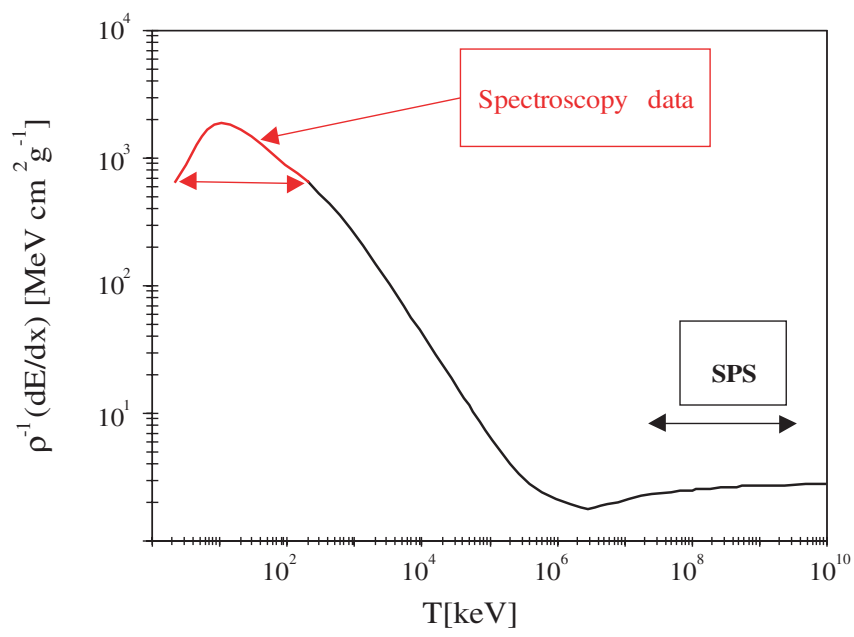

FIG. 1. (Color) Energy loss scaling from the energy range explored in spectroscopy to the SPS one. 
TABLE I. Experimental beams characteristics.

\begin{tabular}{lcc}
\hline \hline Type & Protons & $\begin{array}{c}\text { Ions } \\
\text { Fully stripped lead } Z=82\end{array}$ \\
\hline Current & $0.14 \mathrm{~A}$ & $0.5 \mathrm{~mA}$ \\
Energy & $14 \mathrm{GeV}$ injection & $180 \mathrm{GeV} / \mathrm{a} . \mathrm{m} . \mathrm{u}$ \\
Injection/extraction & $450 \mathrm{GeV}$ extraction \\
Beam sizes(s) at BPL location (horizontal plane) & $\sim 7 \mathrm{~mm}$ injection & Less than $0.8 \mathrm{~mm}$ \\
\hline \hline
\end{tabular}

But, in the framework of the LHC project, the expected beam densities are so high ( $0.5 \mathrm{Amp}$, rms size $\sim 1.2 \mathrm{~mm}$ ) that an experiment was devoted to the study of the luminescence to be used in a beam profile monitor. Experimental confirmation was first obtained using the SPS proton and heavy ion beams [8]. Beam profiles were obtained and their sizes calculated for different types of beams whose characteristics are given in Table I.

This article summarizes the activities carried out in this framework, giving the measurement results for beam profiles and the monitor resolution estimation for the LHC beam. The results of the experiments performed to determine the main parameters of the luminescence of the nitrogen molecule will also be illustrated.

\section{RESOLUTION: THEORETICAL BACKGROUND}

In the LHC the luminescence monitor is supposed to measure beam sizes along the acceleration cycle. This is possible since, along the beam trajectory, light is emitted whose intensity is proportional to the local beam density. The projection of the luminescent volume in the direction of propagation provides the beam profile and beam size. Many different factors contribute to the monitor resolution.

\section{A. Photon radiation}

Residual gas luminescent emission inside the vacuum chamber can be represented as a two-stage process: excitation by the beam and deexcitation with consequent radiation emission. Both affect the beam profile resolution, the first as an effective radiation source, the second due to the drift of the molecule during the decay time. The first stage can be analyzed by means of the virtual photon method [9] since it meets the validity requirements [10]. The electric field associated with a relativistic particle can be seen as a radiation pulse in the laboratory frame which, in the relativistic approximation, has a disk shape with radial polarization. This can be considered as a bunch of virtual photons traveling at the same velocity as the particle. The virtual photon spectrum has been calculated both in the impact parameter [9] and in the transverse momentum domain [11] and the two representations are related by the Fourier transform:

$$
\begin{gathered}
\frac{\mathrm{d} N}{\mathrm{~d} \omega \mathrm{d} b}=\frac{q^{2}}{\pi^{2} c \hbar \beta^{2}} \frac{\omega}{\gamma^{2} v^{2}}\left[K_{1}^{2}\left(\frac{\omega b}{\gamma v}\right)+\frac{l}{\gamma^{2}} K_{0}^{2}\left(\frac{\omega b}{\gamma v}\right)\right] \\
\frac{\mathrm{d} N}{\mathrm{~d} \omega \mathrm{d} \bar{k}_{\perp}}=\frac{q^{2}}{\pi^{2} c \hbar \omega} \frac{\bar{k}_{\perp}^{2}}{\left[\bar{k}_{\perp}^{2}+\left(\frac{\omega}{\gamma c}\right)^{2}\right]^{2}},
\end{gathered}
$$

where $b$ is the impact parameter, $v$ the particle velocity, $q=e Z$ the particle charge, $\beta$ and $\gamma$ the usual relativistic quantities, $\omega$ the energy and $k$ the momentum of the virtual photon and $K_{n}$ indicates the modified Bessel functions. So (1) can be considered as a white light pulse where the spectrum cutoff depends on the impact parameter $b$. These photons excite the molecular system inducing an electronic transition from the initial state $i$ to the final state $f$ and the number of primary products is proportional to the oscillator strength of the transition $f_{i, f}=\langle f|Q| i\rangle$ where $Q$ is an operator. The second stage of the process, the radiative deexcitation, is a more complex phenomenon because the excited molecule can decay and quench in different ways. Essentially the processes can be differentiated depending on the excitation levels and transitions involved, but for our purpose it is important to distinguish only between (i) deexcitation directly to the ground state and (ii) deexcitation through intermediate states. In (i), only a transition to the ground state is allowed, the transition is fast $\sim 10^{-9} \mathrm{sec}$ and the molecule cannot dissipate vibrational energy in the short transition lifetime. In (ii), the ground state is reached after subsequent allowed transitions. Their lifetime ranges from $10^{-8}-10^{-7} \mathrm{sec}$. This is the case that concerns our measurements.

\section{B. Beam size resolution}

In the image quality field, the resolution is defined by two main characteristic functions correlated by a Fourier transform in the impact parameter domain: the point spread function (PSF) and the modulation transfer function (MTF). The first represents the two-dimensional intensity response to a point source. Its convolution with the geometrical function describing the real source gives the image broadening due to all the possible causes such as diffraction, optical aberrations, detector resolution, etc. The MTF was introduced in the transverse angular spectrum representation $\left(k_{x}, k_{y}\right)$ as the two-dimensional Fourier 
transform of the PSF. In this case, the convolution process is a simple product. The study of the profile quality is reduced to the determination of the PSF, or at least of its rms sizes, to evaluate the resulting deterioration of the real image.

Each aspect of the measurement chain, from the luminescence physical characteristics to the electronic image acquisition chain, can affect the final PSF. The resolution associated with the luminescence monitor has been estimated, in the case of LHC beams, taking into account different factors: (i) the equivalent size of the exciting source; (ii) thermal motion of the ions and momentum exchange during ionization; (iii) ion drift due to the beam space charge force; (iv) ion trapping.

\section{The equivalent size of the exciting source}

Luminescence is produced when a virtual photon associated with a relativistic particle of the beam is exchanged with a molecule (II A). This implies that the pointlike particle producing luminescence has to be considered as an extended source, described in impact parameter domain by (1). This induces a deterioration of the PSF. The corresponding MTF function can be extracted from (2). Owing to the asymptotic behavior of modified Bessel functions the formula (1) has a $b^{-1}$ law up to a cutoff $b_{\max }=\gamma v / \omega$ from which it goes quickly to zero with an $e^{-b} / \sqrt{b}$ dependence. $b_{\max }$ can then be considered as the maximum broadening to the real image due to the extended source. At the LHC maximum energy $(\gamma=7462)$, and emission close to $400 \mathrm{~nm}, b_{\max }$ is $\sim 450 \mu \mathrm{m}$. Nevertheless, if $\langle b\rangle=$ $\int_{h}^{b_{\max }} b f(b) d b / \int_{h}^{b_{\max }} f(b) d b$ is calculated, where $f(b)$ is the function describing the source in the variable $b$, the $b^{-1}$ dependence cuts the broadening rms value down to a few microns, which is negligible compared with the $\sigma \sim$ $1 \mathrm{~mm}$ size of the LHC beam. In the integral $h=b_{\min }$ is a lower limit imposed by the discontinuity at the origin of the Bessel function. It is reasonable to take $h=1 / k_{\perp \max }=$ $1 / k=c / \omega$, since $b$ and $1 / k_{\perp}$ constitute a Fourier couple.

\section{Thermal motion of ions and momentum exchange during ionization}

Thermal motion and momentum exchange effects on the image broadening can be evaluated following Ref. [12]. The average thermal speed can be calculated by means of

$$
\mathrm{v}_{\mathrm{rms}}=\sqrt{\frac{3 R T}{M}},
$$

where $R$ is the universal gas constant, $M$ the molecular mass, and $T$ the system temperature. For $T=293$ Kelvin, a speed $v_{\text {rms }} \sim 0.510^{-3} \mathrm{~mm} \mathrm{~ns}^{-1}$ is estimated. Assuming that the emission probability is constant during the decay time of $60 \mathrm{~ns}$, the result is a drift of $30 \mu \mathrm{m}$. As far as the momentum exchange is concerned, one should refer to [12] in which the maximum contribution is estimated to be around twice that due to the thermal motion. In any case, these factors are reduced when taking into account the decay time and the directive optical aperture effect (i.e. particles whose velocity is parallel to the optical axis do not contribute to image broadening) averaging, respectively, on $\left\langle e^{-\tau x}\right\rangle$ and $\langle\cos x\rangle$. Total calculated averages give a $\sim 0.3$ multiplication coefficient that reduces the $v_{\text {rms therm }}$ and $v_{\text {rms ion }}$ contributions to, respectively, 10 and $20 \mu \mathrm{m}$. Adding these contributions in quadrature an rms broadening of $\sim 22.4 \mu \mathrm{m}$ is obtained.

\section{Ion drift due to the beam space charge force and light emission probability}

As far as the space charge force effect on the emitting molecule is concerned, its broadening contribution can be estimated. Since these effects may play an important role because of the high space charge fields in the LHC beam, a simulation of the nitrogen ion drift due to the space charge force was performed. For simplicity, the initial spatial distribution of the ions was supposed to be uniform, whereas the thermal and momentum exchange velocity were given a Gaussian distribution to define the initial velocities. Assuming an LHC round Gaussian beam with a longitudinal Gaussian distribution in the $z$ coordinate, the space charge electric field was modeled as [13]

$$
E_{r}=2 \frac{N_{p} r_{e} m_{e} c^{2}}{e} \frac{e^{-\left(z^{2} / 2 \sigma_{z}^{2}\right)}}{\sigma_{z}} \frac{1-e^{-\left(r^{2} / 2 \sigma_{r}^{2}\right)}}{r},
$$

where $e$ is the electron charge, $m_{e}$ its rest mass and $r_{e}$ its classical radius, $N_{p}$ the number of particles, and $r$ and $z$ the transverse and the longitudinal coordinates. The simulation was performed within a temporal window of $100 \mathrm{~ns}$, roughly the double of the 60 ns decay time, to be conservative. Furthermore, severe LHC beam characteristics were assumed, i.e. $210^{11}$ protons/bunch, $7 \mathrm{TeV}$ energy, and beam size $\sigma=600 \mu \mathrm{m}$. Results are shown in Figs. 2(a) and 2(b). Figure 2(a) displays the final total ion displacement with respect to the initial positions and Fig. 2(b) the exponential average, taking into account the emission probability delay due to the measured decay time (see IV C 4). The simulation was carried out by integrating the equations of motion of the ions in steps of $1 \mathrm{~ns}$.

In Fig. 2(a) it can be noted that the displacement distribution is peaked around $0.8 \mathrm{~mm}$ even though its rms value is $\sim 0.65 \mathrm{~mm}$. It is so evident that gases with very short decay time should be preferred reducing the ion displacement during the emission. This is confirmed if we take into account the nitrogen decay time ( $\sim 60$ ns) [see Fig. 2(b)]. This is the real source contribution to the image broadening resulting from the total radiative process. In this case the source is not Gaussian shaped and the only real resolution contribution can be obtained by convolution with the beam profile. Here are some estimates: its FWHM value is $\sim 70 \mu \mathrm{m}$ with much larger drifts in the low density tails 


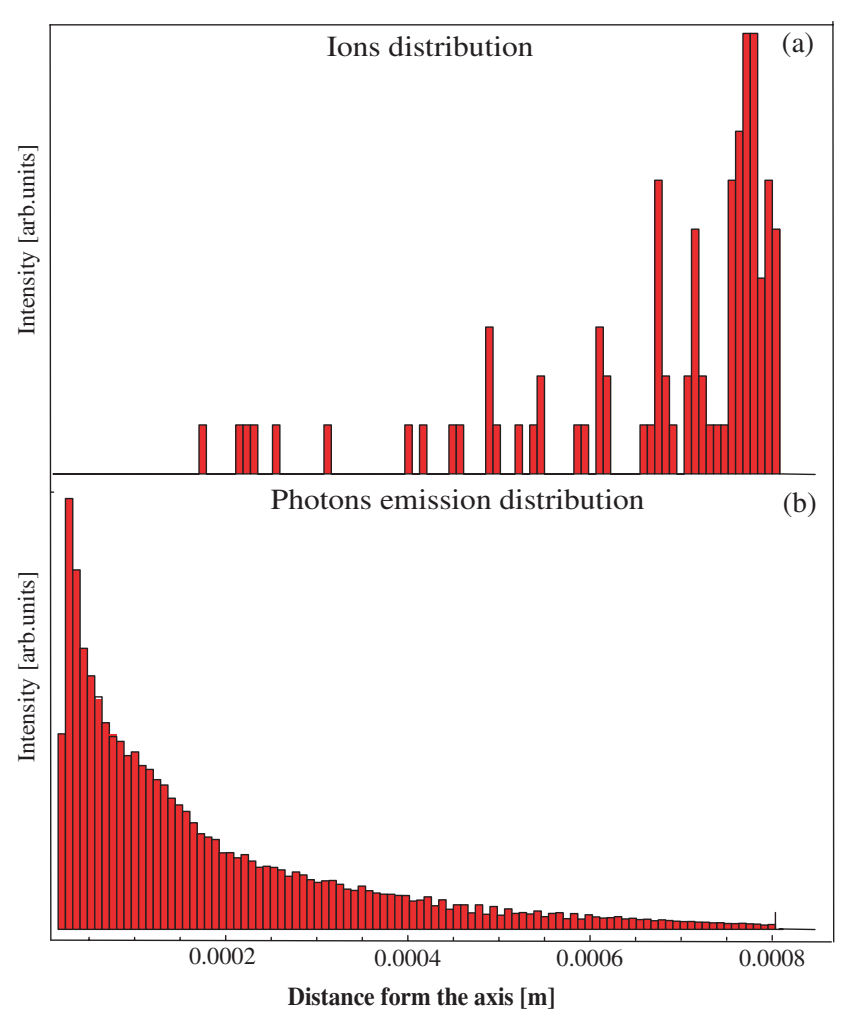

FIG. 2. (Color) Ion drift simulations results. (a) The ions are radially accelerated by four LHC bunches. Their location after $100 \mathrm{~ns}$ is displayed. (b) For each ion step a different probability of light emission is taken into account following the decay time. This provides the distribution of the photon emission.

which can reach a value of $\sim 400 \mu \mathrm{m}$ at $4 \sigma$. These values do not drastically affect the LHC beam measurements.

This simulation was validated by an experiment [14] performed in the SPS where a uniform electrostatic electric field of $\sim 60 \mathrm{kV} / \mathrm{m}$ was applied across the beam pipe. Under its effect the ions drift in the vacuum chamber and give a displacement of the luminescent emission (see IV C 4). The measured displacement of $\sim 0.3 \mathrm{~mm}$ is in very good agreement with the simulated one (the difference is less than $\sim 5 \%$ ).

\section{Ion trapping}

Ion trapping due to the beam potential is supposed to influence the ion distribution in the internal part of the beam itself [15]. In our case the problem does not exist in the proton beam, since the collected light is emitted by positive ions that are repelled by protons. However, this effect could be visible and has to be considered if negative ions are chosen to produce luminescence. Nevertheless, permanent ion trapping in the beam could be avoided [16] by choosing a gas that meets the condition:

$$
A<N_{b} r_{p} L_{\mathrm{sep}} / 2 \sigma y(\sigma x+\sigma y)
$$

(with $A=$ atomic mass, $\mathrm{Nb}=$ number of particles per bunch, $L_{\text {sep }}=$ separation between bunches, $\sigma x, \sigma y=$ horizontal and vertical rms beam size).

In our opinion these are the only effects that could deteriorate a profile measurement due to space charge effects as, once the photons are emitted, no beam related effect can influence them anymore.

\section{THE MEASUREMENT SETUP: THE BPL MONITOR}

The experiments on luminescence produced in the SPS ring by ultrarelativistic proton and ion beams were carried out in the beam profile with a luminescence (BPL) monitor whose setup is given in Fig. 3. For fixed target experiments, the PS injects the beam in two batches at $14 \mathrm{GeV}$ in the SPS where it is further accelerated to the extraction energy of $450 \mathrm{GeV}$. During the acceleration ramp the unnormalized emittance shrinks as a consequence of adiabatic damping. This produces a reduction of the beam sizes in both horizontal and vertical directions by a factor of around six. The SPS revolution frequency is $44 \mathrm{kHz}$ with bunches of $3 \mathrm{~ns}$ length separated by $2 \mathrm{~ns}$. The average circulating current is 0.14 A, i.e., beams of $\sim 2 \times 10^{13}$ protons.

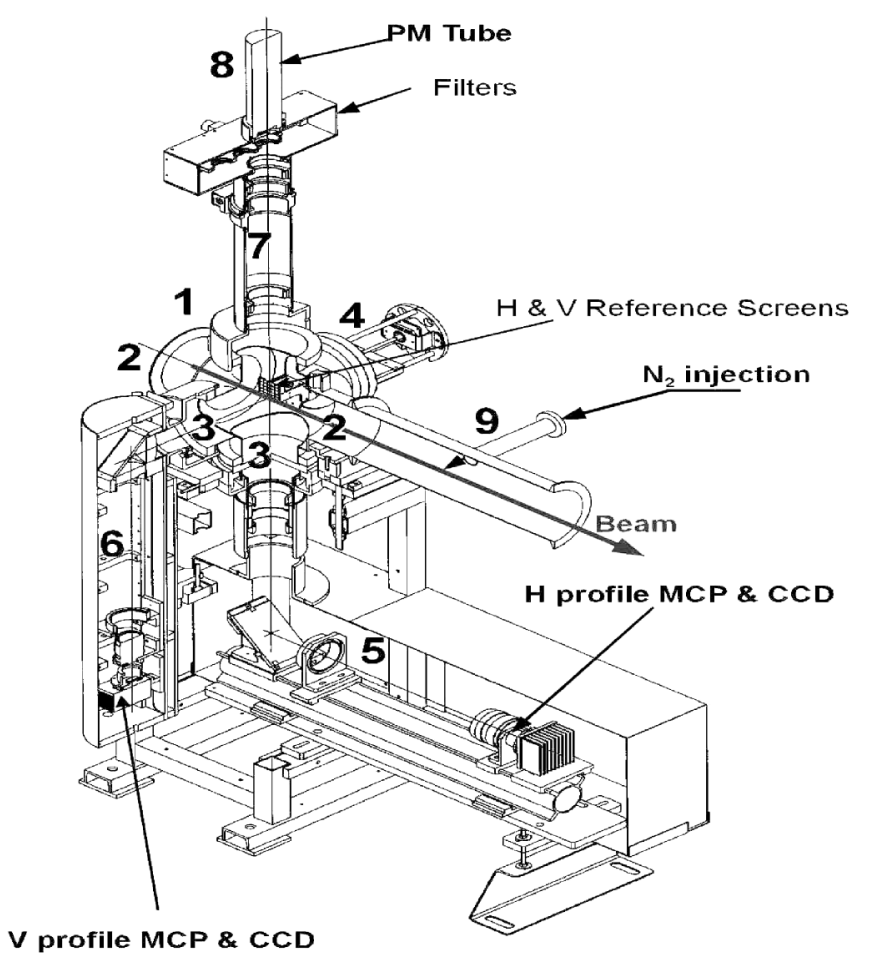

FIG. 3. (Color) The BPL experimental setup installed in the SPS ring: (1) vacuum tank, (2) the ports dedicated to the beam passage, (3) $\mathrm{H}$ and $\mathrm{V}$ optical windows for light extraction and beam imaging, (4) calibration reference screens, (5) $\mathrm{H}$ profile optical setup, (6) V profile optical setup, (7) optical channel for the counting experiment, (8) photomultiplier, (9) gas injecting channel. 


\section{A. Mechanical setup}

Basically the BPL is made up of a 450-mm long six-port vacuum tank with 160-mm diameter tubes (1) (see Fig. 3). Two ports are used for the beam (2), three are equipped with $144 \mathrm{~mm}$ quartz windows for measurements (3), and the last one has a remote controlled reference screen for optical calibrations (4). Close to this tank there is a nitrogen injection system (9) and a local pressure measurement gauge. The three optical setups [(5), (6), (7)] are protected from parasitic light. The horizontal profile channel ends with an optical rail providing many options for optical experimentation. It allows to shift the camera with respect to the other elements. The vertical setup is under a primary vacuum and points to the floor. Optical elements are fixed and previous laboratory setups were necessary. The photomultiplier (PM) channel is also fixed and is equipped with a remote controlled diaphragm positioned between two lenses. Its distance from the first lens is equal to the focal length in order to study angular emission. The whole setup is simple, inexpensive, and very reliable as it does not make use of any component under ultrahigh vacuum (UHV).

\section{B. Optics}

Following the quartz windows three different optical transport lines collect a maximum of emitted photons and produce a beam image (see Fig. 3). The first is dedicated to the horizontal beam profile (5), the second to the vertical one (6), and the third (7) provides a horizontal image on a photomultiplier (8) for photon counting to perform cross section and decay time measurements. All systems use $80 \mathrm{~mm}$ diameter achromatic lenses of various focal lengths to maximize the collected flux and to minimize chromatic and spherical aberrations. On all the optical channels, the insertion of chromatic filters was foreseen. These configurations were calculated by means of an optical ray-tracing code [17] and tested before installation. The horizontal setup was designed to concentrate the flux on few pixels thus allowing a higher photon density for large beams at the beginning of the acceleration ramp. Furthermore, it meets the need to locate the image plane (corresponding to the camera position) far enough from the beam plane to be protected from background particles. This results in a magnification ratio that gives a scaling of $285 \mu \mathrm{m} /$ pixel, even though the strong object reduction results in a deterioration of the resolution. Another optical system was installed to measure vertical profiles. In this case, since the vertical beam size is smaller, the scaling was reduced to $180 \mu \mathrm{m} / \mathrm{pixel}$, thus allowing a better imaging performance. In these tests the different magnification ratios between horizontal $(\mathrm{H})$ and vertical (V) optical channels were particularly suited as $\beta_{h}$ is nearly twice $\beta_{v}$ and the beam sizes shrink during the acceleration cycle. As far as the first setup is concerned, different measurements of the modulation transfer function

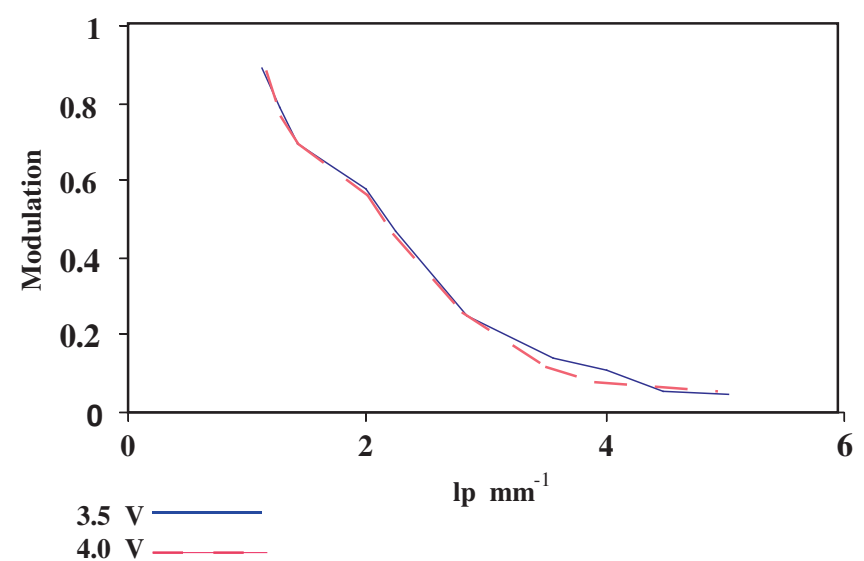

FIG. 4. (Color) MTF measurements of the horizontal optical setup for different MCP gains. The stability of the MTF is noteworthy.

were performed to determine the limiting resolution while varying the intensifier gain. An example for two different gains is shown in Fig. 4 (lower applied voltages $=$ higher gain, see Fig. 10).

It should be noted that the limiting contrast is fixed at $0.05 \%$ at $\sim 5 \mathrm{lp} / \mathrm{mm}(\sim 200 \mu \mathrm{m})$. The rms resolution is affected by the drastic fall of the MTF spectrum and its value is $\sim 2-2.5 \mathrm{lp} / \mathrm{mm}(400-500 \mu \mathrm{m})$ which added in quadrature can produce a $20 \%$ broadening of a $0.6 \mathrm{~mm}$ rms beam (minimum size measured in the nearest wire scanner in the SPS ring at $450 \mathrm{GeV}$ ). A better performance was obtained in the vertical setup where the estimated rms resolution $(200-250 \mu \mathrm{m})$ produces a $3 \%-4 \%$ image broadening for the above-mentioned beam. For the crosssection measurements, the beam is imaged by means of a telescope onto the photomultiplier active surface. So it was necessary to measure the efficiency of the PM for points at different distances from the optical axis and for different angles of incidence. This was performed with a blue LED

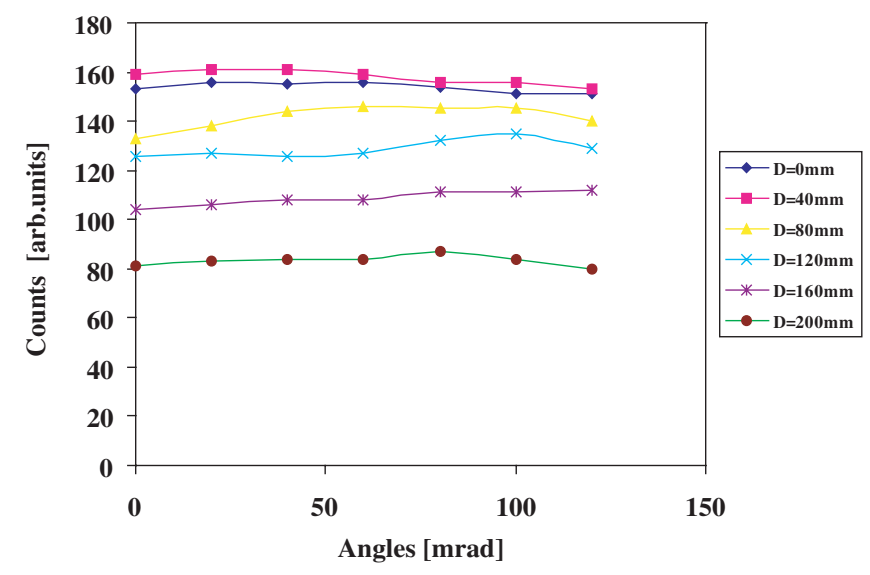

FIG. 5. (Color) PM efficiency as a function of the position and of the angle of incidence of the blue LED light on the PM active surface. 
and a system of diaphragms to simulate a directive source. The results are plotted in Fig. 5 where a difference in efficiency of $\sim 50 \%$ is noticed with a light spot at $20 \mathrm{~cm}$ from the center. With regard to the efficiency as a function of the impinging position, the decrease of the PM response for marginal points is noticeable.

For each different incidence point different impinging angles ranging from 0 to $0.12 \mathrm{rad}$, that is the aperture of the telescopic system, were also tested. The angular dependence was determined for different incidence points on the sensitive surface and the angular response can be considered constant over the whole range.

\section{Detection and acquisition}

In the $\mathrm{H}$ and $\mathrm{V}$ image plane, the light is collected onto a DEP PP0360R two-stage intensifier fiber-optically coupled to a Peltier cell cooled CCD TH7863. A reduction of 50\% of the thermal signal is achieved when decreasing the CCD temperature by 8 degrees. The beam image is acquired by an 8 bit video frame grabber on a 14 bit slow scan digitizer, both in the Versa Module Eurocard (VME) standard. The acquisition rate of the frame grabber $(20 \mathrm{~ms})$ allows one to scan the energy ramp (14-450 GeV) with an accuracy of $\sim 1 \mathrm{GeV}$ since the acceleration rate is $1 \mathrm{GeV} / 25 \mathrm{~ms}$. Local data processing performs a Gaussian fit on the beam projection; beam images in 3D (light density is the 3rd dimension), 2D and projections can be displayed. Numerical filter and Gaussian fit routines have been developed specifically for the luminescence application to reduce the noise. A statistical routine, averaging ten profiles, was made and tested for very low gas pressures and big sized beams (see IVA).

In the channel dedicated to counting experiments, the photon detection and acquisition were performed with an XP2020 photomultiplier with a Bialkali type D SbKCs photocathode and a borosilicate window. The radiant sensitivity is respectively $83 \mathrm{~mA} / \mathrm{W}(391 \mathrm{~nm})$ and $80 \mathrm{~mA} / \mathrm{W}$ $(427 \mathrm{~nm})$ with quantum efficiency of $\sim 26.3 \%(391 \mathrm{~nm})$ and $\sim 23.2 \%(427 \mathrm{~nm})$. Current amplification is estimated to be $2 \times 10^{7}$ with a dark current of 1000 counts/s and a time resolution for the single photon event of $5 \mathrm{~ns}$ FWHM. To distinguish real counts from noise a DC input discriminator was used. Its threshold ranges from $-5 \mathrm{mV}$ to $-150 \mathrm{mV}$ and the width is adjustable from 8 to $100 \mathrm{~ns}$ with a maximum rate exceeding $50 \mathrm{MHz}$ and double event resolution of $\sim 15 \mathrm{~ns}$. Various tests were performed in the BPL setup to determine the plateau of the PM sensitivity in the experimental environment.

\section{Vacuum system and gas injection}

Gas can be injected through a remote controlled leak valve [18]. The pressure bump is restricted in the SPS experiment to the monitor area by two $400 \mathrm{l} / \mathrm{s}$ sputter ion pumps installed $\pm 4 \mathrm{~m}$ from the monitor. Interlocks protect the SPS vacuum system from excessive pressure bumps. A turbomolecular pump, equipped with venting securities in case of a turbo pump fault, performs the preliminary pumping. A safety valve isolates this pump. It can be opened only if the rotation velocity of the turbo pump is higher than $80 \%$ of its nominal velocity $(P<$ $10^{-5}$ mbar). An electromagnetic right angle valve isolates the injection. Its closing is controlled by a cold cathode gauge and by the Penning gauge that checks the machine vacuum. The distance of the vacuum gauges from the interaction region is the principal cause for the uncertainty of the gas pressure. It is very difficult to assess the error, but a rough estimate indicates that a $100 \%$ error in the pressure determination has to be taken into account. The valve system optimizes the gas pressure before injection and checks the injection valve before the machine vacuum valve is opened. The injection valve range is $5 \times$ $10^{6}-10^{13} \mathrm{mbar} \mathrm{l} / \mathrm{s}$ and its sealing is $10^{-9} \mathrm{mbar} \mathrm{l} / \mathrm{s}$. In case of default the valve closing time is 3 seconds which justifies the installation of a second valve interlocked by Penning gauges. The injection valve is operated by a RVC2000 controller and injection is carried out by flux regulation. The vacuum components represent the most expensive part of the BPL system.

\section{EXPERIMENTAL RESULTS}

The SPS experiments were carried out in different stages each performing different tasks. Priority was given to profile measurements with both proton and ion beams. Image quality and its dependence on the different parameters such as pressure, multichannel plate (MCP) gain, and energy were analyzed. The main characteristics of the luminescent emission of nitrogen molecules were measured. The angular dependence of the emitted radiation was also studied. Finally, the gas pressure bump in the vacuum chamber was measured to estimate its influence on beam dynamics.

\section{A. Beam profiles and beam size}

As previously mentioned, the beam, crossing the injected gas pressure bump, produces light in the BPL. The photons are collected and imaged in the CCD where the light is visualized. It is possible to obtain the beam profile by projecting it onto the horizontal axis of the CCD (for both $\mathrm{H}$ and $\mathrm{V}$ setups the camera displays a vertical column allowing fast projections, see Fig. 6).

After the acquisition a standard in-house software tool performs the fit of the profile. The model is a Gaussian curve $=a 1+\left[a 2 \exp \left[-(x-\mu)^{2} / 2 \sigma^{2}\right]\right] / \sigma \sqrt{2 \pi}$. First of all, the routine calculates the rms value and the profile barycenter. These are then taken as starting values for the fit that determines the main parameters $a 1, a 2, \mu$, $\sigma$. Other typical values are assessed, such as the FWHM, the fit variance, and the confidence interval for the fitted parameters. A noise subtraction, whose level is fixed by $a 1$, 

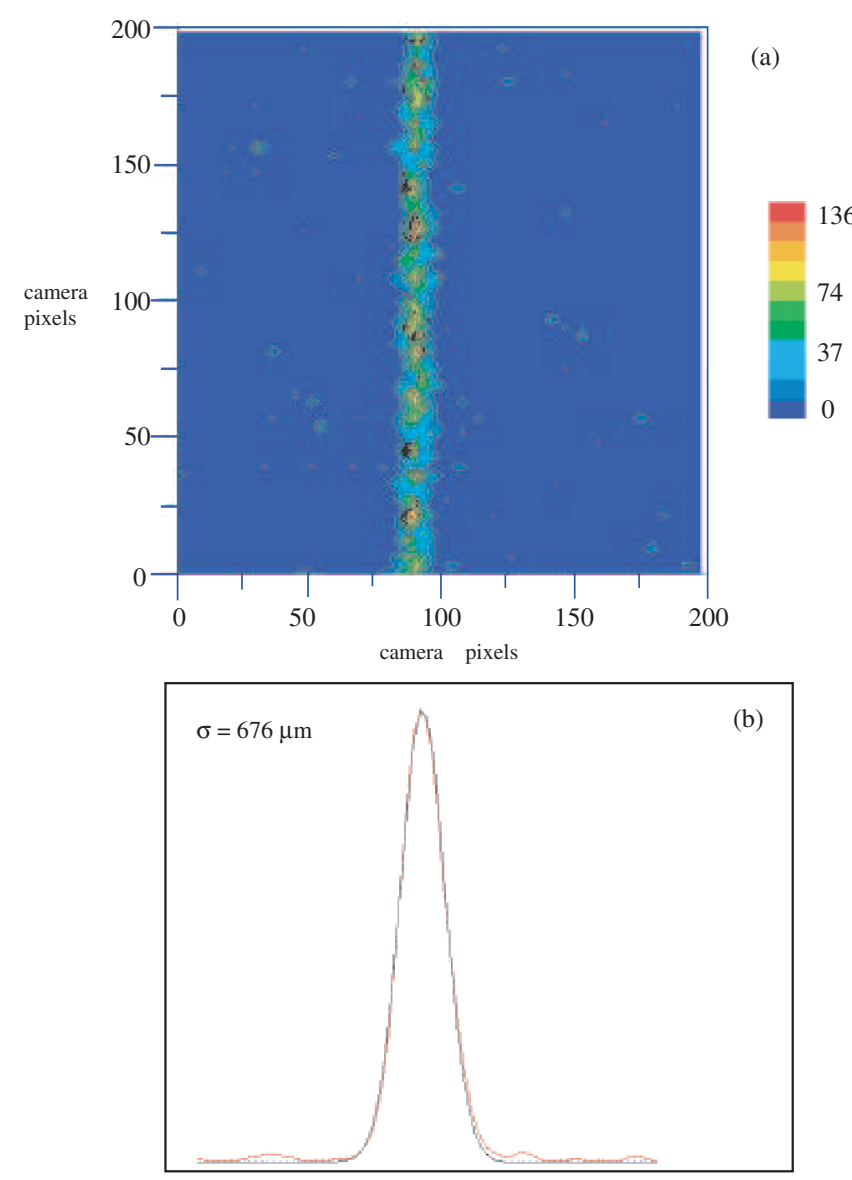

FIG. 6. (Color) (a) Display of the proton beam scintillation path (the display is similar for the $\mathrm{H}$ and $\mathrm{V}$ plane owing to camera orientation). (b) Vertical profile obtained by projection on the horizontal axis on which the result of the Gaussian fit is superimposed. The measurement conditions were beam $\sigma=676 \mu$, beam current $140 \mathrm{~mA}, 2 \times 10^{13}$ protons, beam energy $=$ $450 \mathrm{GeV}$, and nitrogen pressure $=5 \times 10^{-7}$ Torr.

is then performed and the rms calculated again. Many acquisitions were performed and the relative beam size evaluated as a function of different variables. Since the main goal of nondestructive SPS and LHC monitors is to follow the emittance blowup as a function of time and energy, considerable effort was devoted to this kind of measurement. Various images were acquired at different times, corresponding to different energies. In Fig. 7 the beam size shrinking during the whole ramp is illustrated. The stars and the squares indicate, respectively, the fitted sigma and the calculated FWHM value of the beam. In the timing range from 0 to $5000 \mathrm{~ms}$ the energy varies from 14 to $450 \mathrm{GeV}$.

After these relative measurements it was necessary to evaluate the accuracy of the luminescence monitor. This was performed by comparing the BPL measurements with those made by a rotative wire scanner (WS) located 244 meters upstream. The result of this analysis is shown in Fig. 8(a), which illustrates the beam size reduction

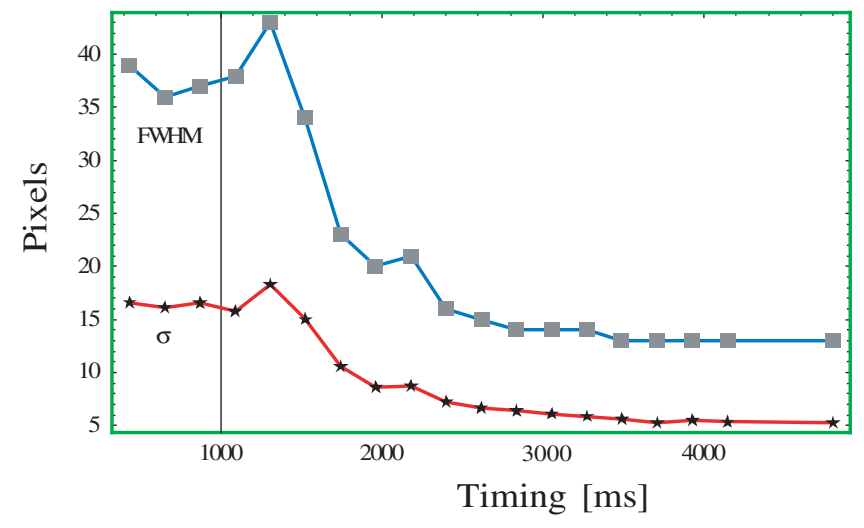

FIG. 7. (Color) Beam sizes evolution during the ramp ranging from 14 to $450 \mathrm{GeV}$.

during the accelerating ramp, measured in the BPL and in the wire scanner.

In Fig. 8(b) the results were normalized to the energy by multiplying them by the factor $\sqrt{\gamma / \gamma_{14 \mathrm{GeV}}}$, to take into
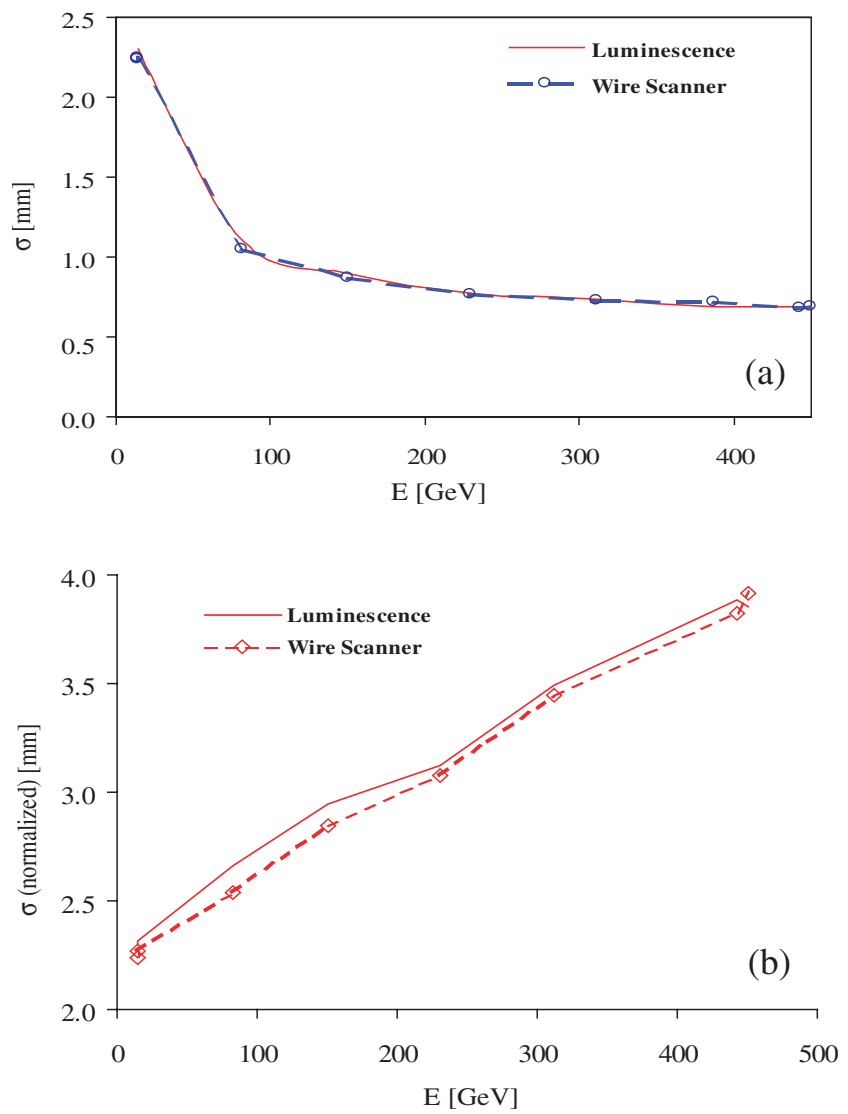

FIG. 8. (Color) (a) Beam size variation vs energy. The continuous and the dotted lines represent, respectively, the BPL and WS measurements. (b) Normalized beam size vs energy during the acceleration ramp. Note that the normalized beam size increases by roughly a factor of 2 which implies an estimated emittance blowup of a factor $\sim 4$. Figure 8(b) was already published in [8]. 
account the adiabatic damping. In this way, the relative emittance blowup during the acceleration ramp can be highlighted. In this case this is estimated to a factor $\sim 4$. The relative increases measured in the WS and in the BPL are in excellent agreement. It should be mentioned that the agreement among measurements implies a slight disagreement with the expected theoretical values since the beam size in the BPL should scale as the square root of the beta function ratio corresponding, respectively, to the WS and the BPL locations. This ratio establishes the accuracy of the BPL with respect to the reference wire scanner at an estimated $17 \pm 2 \%$. Unfortunately, this is also the order of magnitude of the uncertainty of the beta functions in the SPS. To evaluate the absolute accuracy of the BPL a wire scanner should be installed in its immediate vicinity.

The horizontal setup was used mainly to test the imaging performances at low energies (where the larger beam sizes are not influenced by resolution deterioration) and very low pressure.

As mentioned in Fig. 6, very good results were obtained at $5 \times 10^{-7}$ Torr. Irrelevant image deterioration was noted when reducing the nitrogen pressure to $10^{-7}$ Torr. Below this limit the poor counting statistics result in a bad signal to noise ratio and a beam signal modulation at the limit of detection. To further reduce the nitrogen pressure, while maintaining the image quality (for beams with an rms size of the order of a few millimeters), a statistical method for image acquisition and analysis was developed. A number of routines following a given procedure are carried out: (i) several images (matrices) are acquired in successive cycles (basically, ten were used); (ii) the average image is calculated and the profile is obtained by means of a projection on the horizontal axis; (iii) a Gaussian fit is applied by minimizing the chi square function; (iv) once the average noise level is obtained from the fit, it is subtracted from the profile having an effect of noise filtering; (v) another Gaussian fit is applied to the resulting profile, thereby obtaining fit parameters, rms and FWHM values.
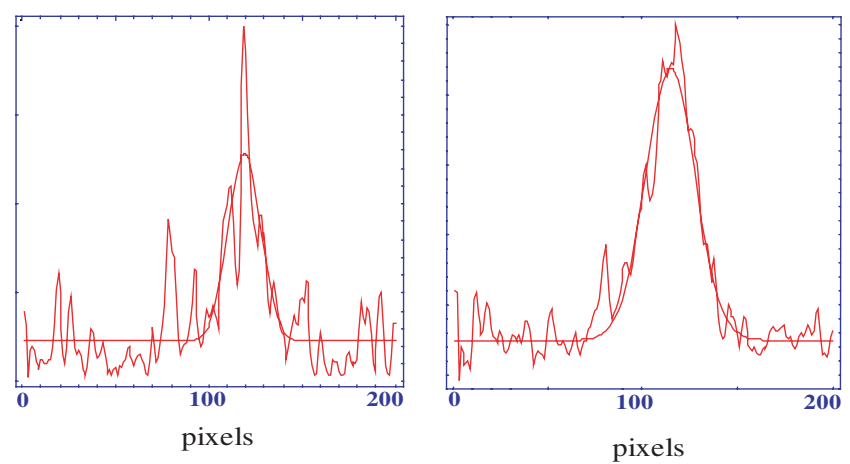

FIG. 9. (Color) Beam profile acquisitions with nitrogen pressure $=5 \times 10^{-8}$ Torr. Beam current $=140 \mathrm{~mA}$, energy $=14 \mathrm{GeV}$, and $\sigma \sim 4.7 \mathrm{~mm}$. The Gaussian fit is superimposed on the experimental data.

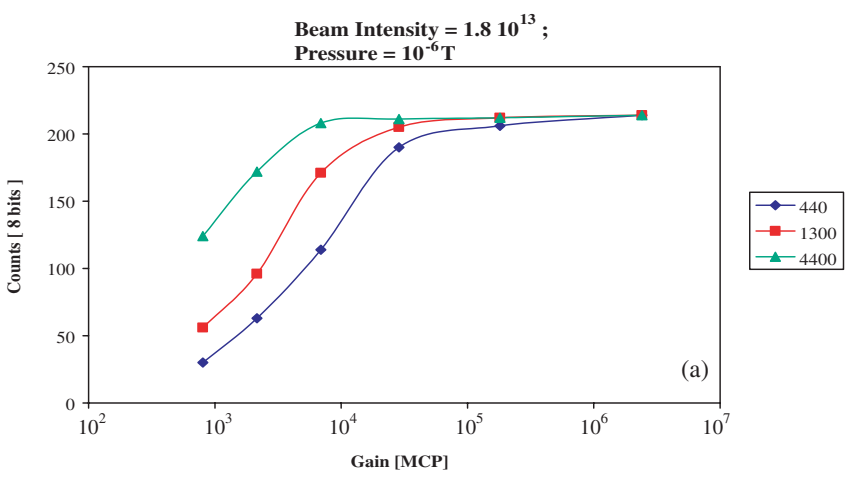

FIG. 10. (Color) CCD counting vs MCP gain for different beam sizes. They decrease as a function of the timing event in the ramp ( $T=440=$ larger sizes, $T=4400=$ smaller sizes). The figure displays the count variation versus the MCP gain.

Figure 9 shows a result obtained for the first $14 \mathrm{GeV}$ beam injection. The profiles with the superimposed Gaussian fit can be seen: on the left a single acquisition profile, on the right the statistically processed one. In the latter the image quality is clearly much higher and the fitted results give a measurement consistent those at $10^{-6}$ Torr (better than 10\% accuracy), whereas the former shows a sigma error in the order of $40 \%$.

The beam profile acquisition, and the subsequent sigma determination, also allowed the analysis of the BPL performances as a function of different important parameters. The pressure and the MCP gain dependence were analyzed. Different measurements were carried out varying the pressure from $5 \times 10^{-8}$ to $10^{-6}$ Torr. The stability of BPL results vs pressure was estimated to be in the order of $5 \%$, that is in the range of the beam size fluctuations, as similar results were obtained with the wire scanner.

Other beam size measurements were performed fixing the pressure and varying the MCP voltage. The results indicate that for small beam sizes (i.e. high local beam density) there is a gain range $\left(3 \times 10^{5}-4 \times 10^{6}\right)$ in which saturation occurs depending on parameters such as pressure, beam intensity, beam size, and MCP gain. This can be detected by using the signal integrated on the $\mathrm{CCD}$ as can be observed in Fig. 10 where the CCD signal amplitude is plotted as a function of the MCP gain. It can be seen that for increasing energies, i.e. timing events, saturation occurs at decreasing MCP gains. Consequently, a timing driven voltage ramp, scaled with the circulating beam current, has been applied to the MCP supply to give the proper intensifier gain over the whole SPS cycle.

\section{B. Lead ions}

The BPL was also tested with lead ion beams and produced very good results. In this case the beam intensity is reduced with respect to the proton beam, while a photon flux increase is obtained owing to the $Z^{2}$ scaling. The observed signal to noise ratio is better than with proton 


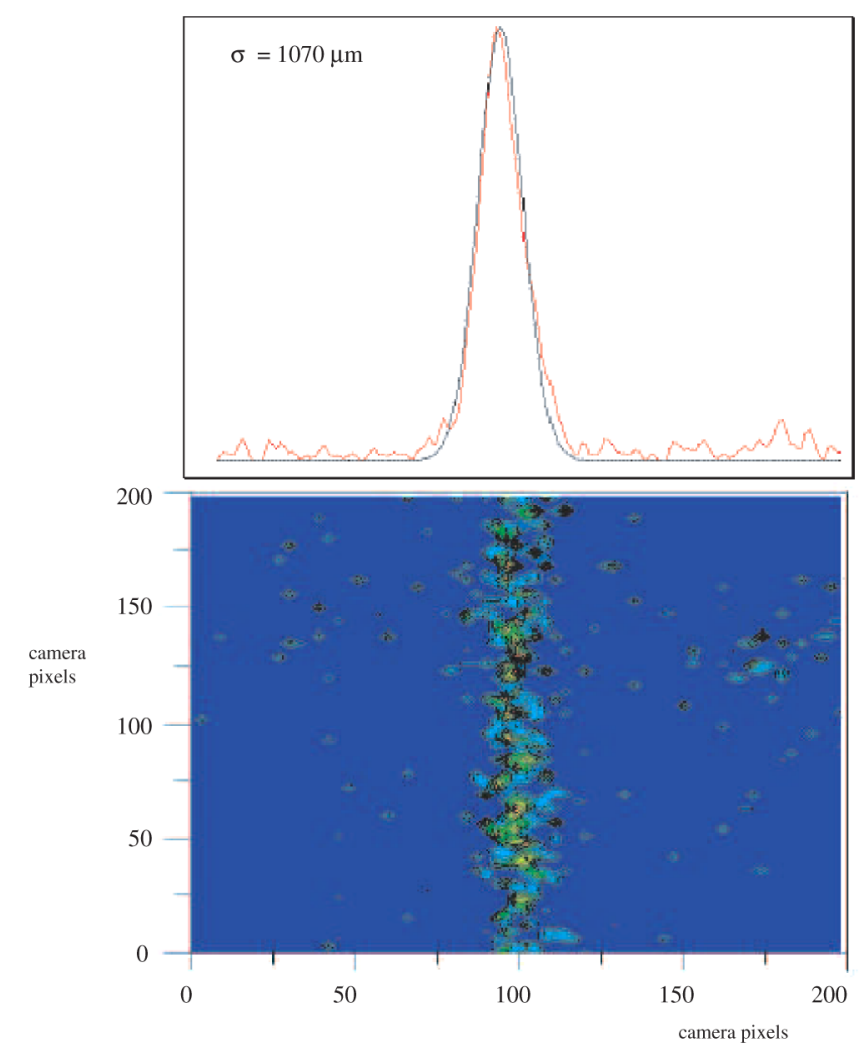

FIG. 11. (Color) Horizontal profile of a beam of fully stripped lead ions. The intensity is $\sim 10^{9}$ ions, $Z=82$ and nitrogen pressure $=6 \times 10^{-7}$ Torr.

beams. An example is shown in Fig. 11: the intensity was $\sim 10^{9}$ ions. With respect to a proton beam an equivalent light flux was collected due to the factor $Z^{2}=6724$. An evident parasitic noise reduction was detected in the heavy ions experiments which has not been investigated yet.

\section{Counting experiments}

After determining the BPL performances for beam imaging, some experiments were carried out to evaluate the main characteristics of the luminescent emission like the cross section, emission spectrum, decay time, afterglow, angular dependence, and the validity of the emitted photon flux scaling according to the Bethe-Bloch formula. This work was performed using the third BPL optical channel ending with the PM in counting mode.

\section{Bethe-Bloch scaling}

In the context of the determination of the absolute cross section, the reference experimental data is available for $200 \mathrm{keV}$ proton beams [4]. In the following, as far as the cross-section measurements are concerned, it was assumed that the dependence of the cross section on the energy has to follow the Bethe-Bloch formula. The low-energy spectroscopy data is not fully in the Bethe-Bloch range, but rather in a domain in which energy loss is given by the Andersen-Ziegler [19] fitting formulas. Therefore a check was made on the stopping power given by the two models at $200 \mathrm{keV}$. The result shows a negligible discrepancy of $1.5 \%$.

The BPL measurements were compared with data obtained by extrapolating the results given in [4] by scaling. An analysis was first devoted to the relative behavior of the counting measurements as a function of the proton beam energy. Normalizing the results to the measurement at $14 \mathrm{GeV}$, the measurements, taken for different energies during the acceleration ramp, show a good agreement between theory and experiment (the maximum discrepancy is less than 15\%). This confirms that the relative cross-section behavior effectively scales according to the Bethe-Bloch formula.

\section{Cross section}

The cross section is the most important parameter to assess the feasibility of a luminescence monitor since it determines the photon flux emitted by the nitrogen molecules excited by the beam. Experimentally the cross section is defined as follows:

$$
\sigma=\frac{N_{\mathrm{ph}}}{K \delta \Psi \lambda},
$$

where $N_{\mathrm{ph}}$ is the number of collected photons, $\sigma$ the cross section, $K$ a coefficient that takes into account the different real experimental contributions, $\delta$ the molecular gas density, $\Psi$ the proton flux, and $\lambda$ the active length of the beam that is collected on the PM surface $(76.9 \mathrm{~mm})$. Following (5) the $\sigma$ value can be obtained by means of the counted number of photons, once experimental conditions such as pressure, proton flux, and active length are determined. In the real experiment, there are factors represented by $K$ that need to be taken into account. This parameter represents the product of different coefficients that assess the contribution given, respectively, by (i) the optical aperture of the system, (ii) the vignetting, (iii) the quantum efficiency of the PM, (iv) transmission in the chromatic filter, (v) transmission in the light extraction windows and in the lenses, and (vi) the PM efficiency as a function of the impact position on its active surface.

The first coefficient takes into account the fact that the entrance pupil collects only a fraction of the emitted photons. This fraction is given by the ratio of the irradiance calculated for the system aperture to that calculated for the whole emission sphere. The system numerical aperture (NA) is determined by the entrance pupil position and diameter brought back to the object plane. In the BPL setup, the aperture is 0.121 and it can be assumed that the lateral extension of the column and of the first lens are smaller than their distance $r$. In this case, and for a Lambertian source, the fraction of the collected flux can be evaluated as the ratio between the spherical surface subtended by a solid angle with aperture $\theta=$ NA and radius $=r$, and the surface of the whole sphere. 
So in the telescopic system $K_{1}=0.00365$.

The coefficient due to the vignetting has been evaluated by simulating the optical system and its value is $K_{2}=$ 0.49 .

The averaged quantum efficiency of the PM at $\lambda=391$ and $427 \mathrm{~nm}$ contributes with a coefficient: $K_{3}=0.256$.

The chromatic filter used in the counting experiment was peaked at $=400 \mathrm{~nm}$ with a FWHM band of $80 \mathrm{~nm}$. Averaging on the two emission lines the transmission coefficient is $K_{4}=0.55$.

Taking into account all the discontinuity surfaces of the lenses and the measured transmission of the quartz window the estimated coefficient is then $K_{5}=0.68$.

The counting PM efficiency as a function of impinging photon positions and angles has been measured (Sec. III B). The corresponding coefficient is $K_{6}=0.63$.

Finally the experimental correction coefficient is given by the product $K=K_{1} K_{2} K_{3} K_{4} K_{5} K_{6}$. The crosssection experiment was performed with $\Psi=1.025 \times$ $10^{15}$ protons $/ \mathrm{sec}$ and a pressure $\sim 9.4 \times 10^{-7}$ Torr. Photon counting was performed during an integration window of $1 \mathrm{~ms}$ which corresponds to an energy resolution of $40 \mathrm{MeV}$ during the SPS energy ramp. When the chromatic filter is used only the two lines at 391 and $427 \mathrm{~nm}$ have to be considered. Ten acquisitions were averaged to obtain the final result and associated errors estimation. Systematic errors where assessed acquiring the background signal, with the PM entrance diaphragm closed, both with and without beam. Also other systematic effects were evaluated and it turned out that, by far, our main error source is the uncertainty on the nitrogen pressure value whose total error estimation is around $100 \%$. The cross-section measurement results for different beam energies are given in Table II, column 3.
The results show a discrepancy with the expected theoretical prediction in column 1 . In fact, measured cross sections are smaller by a factor four to five than the calculated ones. These results have a good agreement with those obtained at lower energy in the PS [7]. In that case a 6.7 reduction factor in respect to the $200 \mathrm{keV}$ data was observed. Different hypotheses could be put forward to explain this difference. First of all, it should be noted that not only do experimental results contain systematic errors, but also theoretical estimates are based on basic assumptions. In Table II, column 1, the cross sections are scaled by means of the Bethe-Bloch equation in the form provided in [19] in formula (23.1). A more complex approach to evaluate the lower energy transfer due to escaping knock-on electrons is also suggested in [19]. The formula is changed by introducing an energy cut to be assessed on a case-by-case basis. If, in our case, we assume the energy cut to be the average ionization energy of the $\left(\mathrm{N}_{2}{ }^{+}\right)$molecule $(242 \mathrm{eV})$, then the estimated cross sections are reduced by a factor depending slightly on the energy in our experimental range. Taking this factor into account brings the estimated values closer to the measured ones (factor three). The analytically estimated cross section corrected for the knock-on electrons contributions are also given in Table II, column 2. At this stage the large uncertainty on the nitrogen pressure (see Sec. III D) has to be recalled. Other possible energy-dependent redistribution mechanisms of the energy loss in the molecular gas are possible (for example the energy loss due to nitrogen ionization...), but their analysis is beyond the scope of this article. Another aspect to be taken into account is the polarization and the angular distribution of the emitted radiation that could play a role. This is due to the fact that, even though the molecule's rotation period is in the

TABLE II. Cross section as a function of the beam energy: Bethe-Bloch scaling from [4] in column 1, corrected for the knock on electrons in column 2 , and measurement results in column 3.

\begin{tabular}{lccc}
\hline \hline $\begin{array}{l}\text { Beam } \\
\text { energy }\end{array}$ & $\begin{array}{c}\text { Cross section, scaling from } \\
\text { 200 KeV data }\end{array}$ & $\begin{array}{c}\text { Cross section scaling corrected for } \\
\text { knock on electrons }\end{array}$ & $\begin{array}{c}\text { Cross section } \\
\text { measurements }\end{array}$ \\
\hline $200 \mathrm{KeV}$ & $\ldots$ & $\ldots$ & $3.81 \times 10^{-17} \mathrm{~cm}^{2}$ \\
$14 \mathrm{GeV}$ & $1.41 \times 10^{-19} \mathrm{~cm}^{2}$ & $8.91 \times 10^{-20} \mathrm{~cm}^{2}$ & $3.22 \times 10^{-20} \mathrm{~cm}^{2}$ \\
$50.5 \mathrm{GeV}$ & $1.64 \times 10^{-19} \mathrm{~cm}^{2}$ & $1.03 \times 10^{-19} \mathrm{~cm}^{2}$ & $3.36 \times 10^{-20} \mathrm{~cm}^{2}$ \\
$64 \mathrm{GeV}$ & $1.69 \times 10^{-19} \mathrm{~cm}^{2}$ & $1.06 \times 10^{-19} \mathrm{~cm}^{2}$ & $3.56 \times 10^{-20} \mathrm{~cm}^{2}$ \\
$95 \mathrm{GeV}$ & $1.76 \times 10^{-19} \mathrm{~cm}^{2}$ & $1.10 \times 10^{-19} \mathrm{~cm}^{2}$ & $3.75 \times 10^{-20} \mathrm{~cm}^{2}$ \\
$124 \mathrm{GeV}$ & $1.81 \times 10^{-19} \mathrm{~cm}^{2}$ & $1.14 \times 10^{-19} \mathrm{~cm}^{2}$ & $3.95 \times 10^{-20} \mathrm{~cm}^{2}$ \\
$150 \mathrm{GeV}$ & $1.84 \times 10^{-19} \mathrm{~cm}^{2}$ & $1.16 \times 10^{-19} \mathrm{~cm}^{2}$ & $4.04 \times 10^{-20} \mathrm{~cm}^{2}$ \\
$177.5 \mathrm{GeV}$ & $1.89 \times 10^{-19} \mathrm{~cm}^{2}$ & $1.18 \times 10^{-19} \mathrm{~cm}^{2}$ & $3.86 \times 10^{-20} \mathrm{~cm}^{2}$ \\
$210.7 \mathrm{GeV}$ & $1.91 \times 10^{-19} \mathrm{~cm}^{2}$ & $1.2 \times 10^{-19} \mathrm{~cm}^{2}$ & $3.93 \times 10^{-20} \mathrm{~cm}^{2}$ \\
$250 \mathrm{GeV}$ & $1.94 \times 10^{-19} \mathrm{~cm}^{2}$ & $1.22 \times 10^{-19} \mathrm{~cm}^{2}$ & $3.99 \times 10^{-20} \mathrm{~cm}^{2}$ \\
$299 \mathrm{GeV}$ & $1.97 \times 10^{-19} \mathrm{~cm}^{2}$ & $1.24 \times 10^{-19} \mathrm{~cm}^{2}$ & $3.89 \times 10^{-20} \mathrm{~cm}^{2}$ \\
$328.8 \mathrm{GeV}$ & $1.99 \times 10^{-19} \mathrm{~cm}^{2}$ & $1.25 \times 10^{-19} \mathrm{~cm}^{2}$ & $3.88 \times 10^{-20} \mathrm{~cm}^{2}$ \\
$365.6 \mathrm{GeV}$ & $2.01 \times 10^{-19} \mathrm{~cm}^{2}$ & $1.26 \times 10^{-19} \mathrm{~cm}^{2}$ & $4.05 \times 10^{-20} \mathrm{~cm}^{2}$ \\
$401.6 \mathrm{GeV}$ & $2.03 \times 10^{-19} \mathrm{~cm}^{2}$ & $1.28 \times 10^{-19} \mathrm{~cm}^{2}$ & $3.85 \times 10^{-20} \mathrm{~cm}^{2}$ \\
$425.6 \mathrm{GeV}$ & $2.04 \times 10^{-19} \mathrm{~cm}^{2}$ & $1.28 \times 10^{-19} \mathrm{~cm}^{2}$ & $4.06 \times 10^{-20} \mathrm{~cm}^{2}$ \\
$450 \mathrm{GeV}$ & $2.05 \times 10^{-19} \mathrm{~cm}^{2}$ & $1.29 \times 10^{-19} \mathrm{~cm}^{2}$ & $4.08 \times 10^{-20} \mathrm{~cm}^{2}$ \\
\hline \hline
\end{tabular}


order of $10^{-11} \mathrm{sec}$, a privileged direction of emission can be imposed by the radially polarized excitation imposed during the interaction with the proton bunch. As far as molecular emission is concerned, this is a theoretically complex topic that requires the knowledge of the involved transition level functions and quantum angular momentum number. This theoretical approach goes beyond the scope of this article. However, to check this possibility, some experiments were carried out and the results will be illustrated in the next paragraph.

\section{Angular dependence}

In the telescope setup, a diaphragm was installed in the focal plane of the first lens. In this plane the position variables are transformed into angles at the source. So, by varying the diaphragm aperture photon counting can be performed, while selecting different solid angles of emission from the source. Figure 12 shows the results for different apertures where the results are normalized to the maximum angular acceptance counting. There is a slight different behavior of the two curves. This can be explained by considering the effect of a not fully isotropic emission. Nevertheless the obtained experimental results are not sufficient to explain the discrepancy in the cross section following the Bethe-Bloch scaling.

\section{Decay time and bunch length}

Some time-related measurements were also devoted to study the properties of the luminescence produced by the $\left(\mathrm{N}_{2}{ }^{+}\right)$molecules. This was important to assess their effect on the beam measurements. The first and most important measurement is to confirm that the decay time is a characteristic of the molecule and that the beam energy has no effect. The decay time measurement was carried out by means of the gated photomultiplier. Starting from the

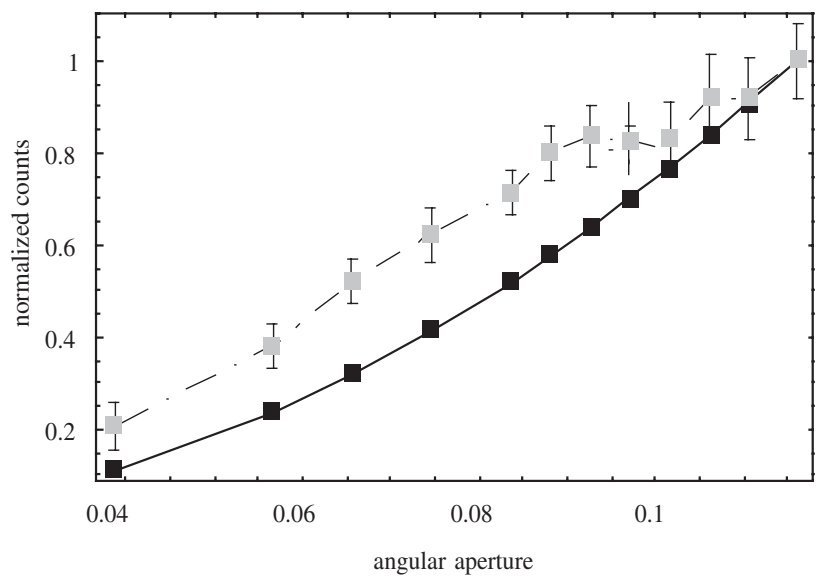

FIG. 12. Counting experiment for different angular apertures. The squares following the continuous line indicate the theoretical values for an isotropical emission. The other points give the experimental data. revolution frequency, a reference pulse was generated by means of a phase locked loop and a programmable delay. The pulse sets a flip-flop $10 \mathrm{~ns}$ before the proton bunch arrival (with an uncertainty of $1 \mathrm{~ns}$ ). The flip-flop opens a gate for a $125 \mathrm{MHz}$ clock to a counter, and the detection of a photon emitted by the proton bunch resets the flip-flop. The counter value is then transferred to the memory whose address is incremented by one. After resetting both the counter and the flip-flop, the system is ready for another acquisition which is performed after a synchronization delay. The results of two typical measurements are shown in Fig. 13. The first measurement was taken with a nominal
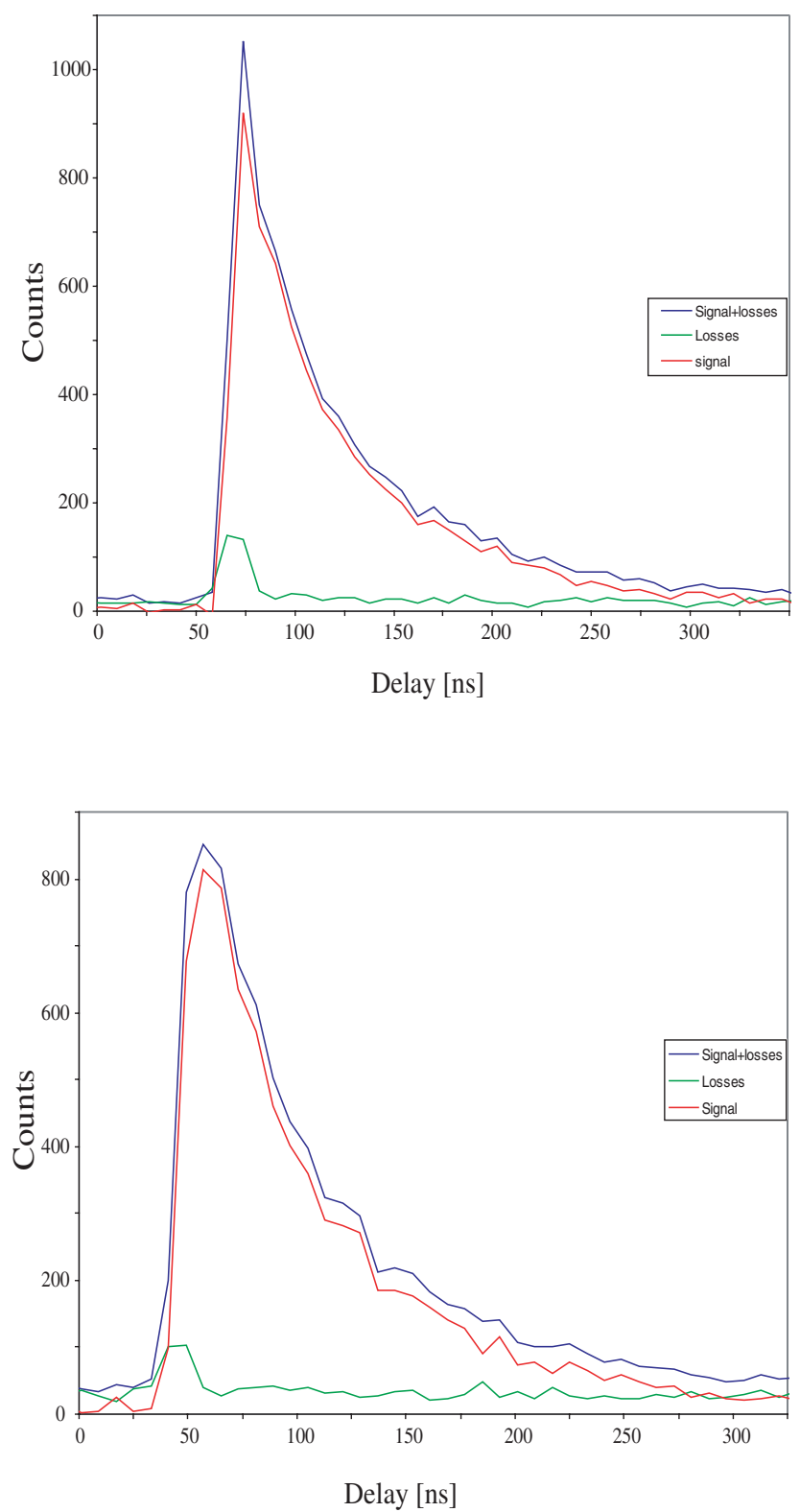

FIG. 13. (Color) Decay time measurements performed with two different bunch lengths. Case 1 is for the nominal bunch; case 2 corresponds to a longer bunch. 


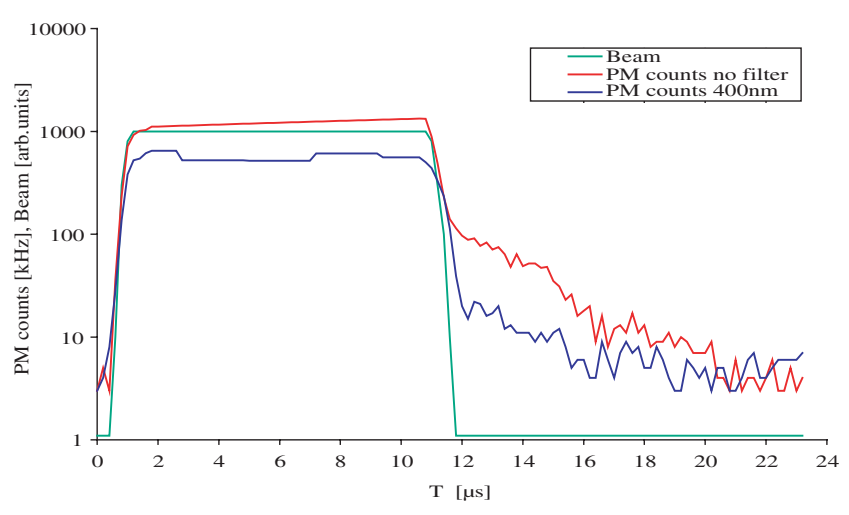

FIG. 14. (Color) Afterglow measurement.

bunch, while the second one was taken during a machine development run where the bunch was longer. The two curves are fitted with a function that is a convolution between a Gaussian distribution (bunch shape) and an exponential decay. The effect of the longer beam is clearly visible in the second case where the exponential behavior is smoothed. The result for the first measurement is a 55.4 ns decay time with a $\sigma=1.4$ ns bunch length. In case 2 the decay time is estimated at $58.2 \mathrm{~ns}$ and the bunch length is equal to $19.5 \mathrm{~ns}$. These results are in very good agreement with the measurements performed at lower energies [7]. The main contribution to the time resolution is given by the delay ( $8 \mathrm{~ns}$ ). The bunch length can be estimated even if it is comparable to the decay time. In the case of short bunches and short bunch spacing, gases with a shorter decay time, such as xenon [7], can be used.

\section{Afterglow}

Another measurement performed with the PM in the telescopic system allowed us to assess the contribution of the afterglow (see Fig. 14). After the beam passage an

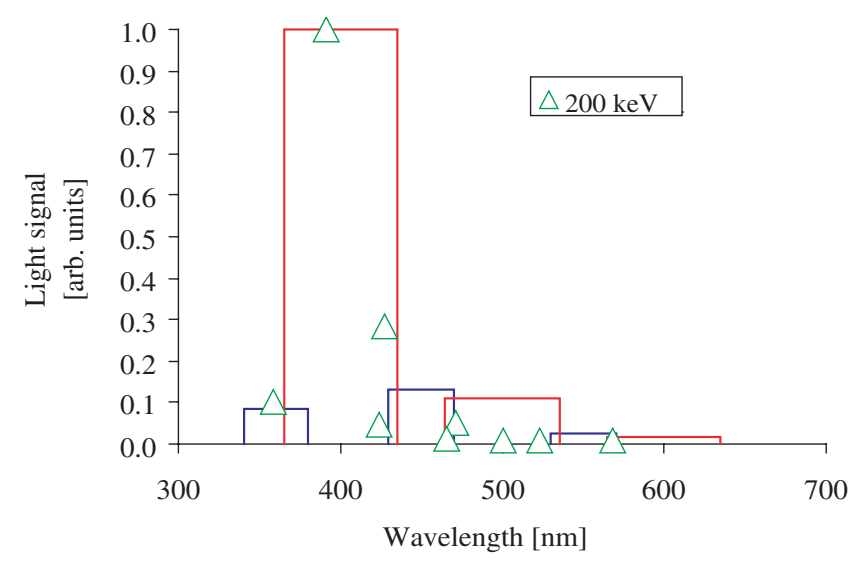

FIG. 15. (Color) Emission spectrum measurement. The boxes represent the relative amplitude measured over the filter bandwidth. The triangles are the spectroscopy results. Figure 15 was already published in [8]

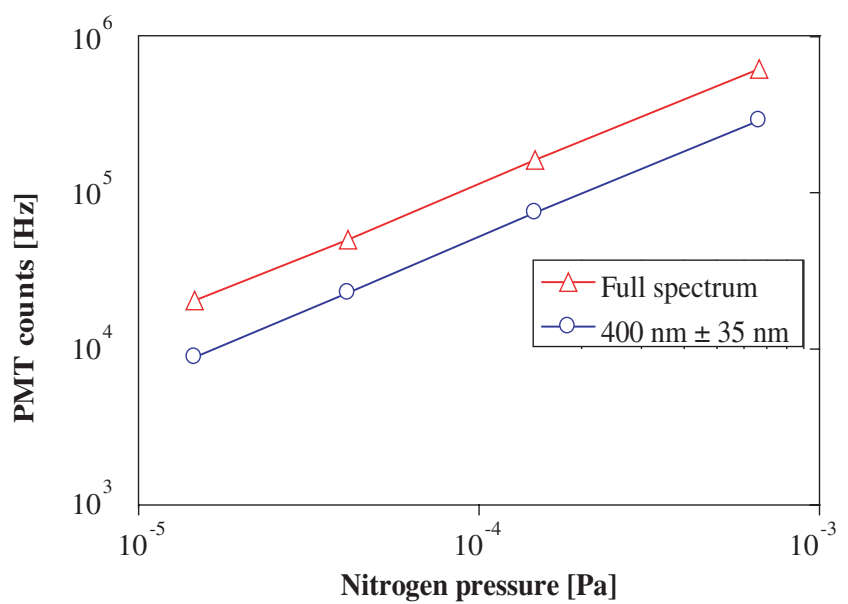

FIG. 16. (Color) Emitted photons vs nitrogen injected pressure. Triangles show the full spectrum acquisition while circles represent the data obtained with a chromatic filter at $400 \mathrm{~nm}$. Figure 16 was already published in [8].

afterglow effect, due to another kind of molecular deexcitation, is evident. This can affect beam measurements if the next beam is separated by less than $8 \mu \mathrm{s}$. Chromatic filters reduce the afterglow contribution (to $4 \mu \mathrm{s}$ ), therefore their use is always recommended when a sufficient photon flux is available.

\section{Emission spectrum and linearity}

To further confirm the low-energy results in the SPS test, the emission spectrum was measured by means of chromatic filters (FWHM passband $=80 \mathrm{~nm}$ ). The result is shown in Fig. 15 where the low-energy data are given too. The relative agreement is excellent and confirms the main emission in the UV-blue range given by the two lines at 391 and $427 \mathrm{~nm}$.

Another important test was performed to confirm the linearity of the number of photons produced as a function of the nitrogen pressure (see Fig. 16) independently of the beam energy. This confirms the assumption that at very low pressure the emission is due to a one-step process and not to recombination. This implies that the emission point is really determined by the impinging particle and no further contribution to the beam profile resolution needs to be considered.

\section{CONSIDERATIONS ON THE USE OF A LUMINESCENCE PROFILE MONITOR IN THE LHC}

\section{A. Expected performances}

In the LHC the beam will be injected at $450 \mathrm{GeV}$ from the SPS in three or four batches of 72 bunches with $1.1 \times$ $10^{11}$ protons each. In the location foreseen for the luminescence monitor, the beta function values are around 
$100 \mathrm{~m}$ ( $x$ and $y$ plane) and the beam size at injection will hence be of the same order as in the SPS at the same energy. It will be possible therefore to perform good measurements, similar to those displayed in Fig. 6, at nitrogen pressures of around $10^{-8}$ Torr. Measurements of individual bunch trains could be considered if the influence of the previous bunch train afterglow is negligible (the separation between batches is $\sim 1 \mu \mathrm{s}$ ). For lead ions, where either 60 or 592 bunches of $10^{7}$ ions will be stored, the situation is similar, i.e., good profiles can be measured at injection at $10^{-8}$ Torr. Once the beams are accelerated, the MCP gain will have to be lowered to take into account the increase of the beam density.

\section{B. Pressure bump and induced radiation estimation}

To consider the use of the luminescence monitor in the LHC, an assessment to determine the effect on the beam of the local pressure increase in the BPL was necessary. For this, the extension of the pressure bump was estimated from the measurements in the BPL setup and the result is shown in Fig. 17.

It can be noted that $2.25 \mathrm{~m}$ away from the injection point the pressure is already reduced by an order of magnitude. So the total average pressure bump can be represented as a square function whose amplitude is the peak pressure with a 4 meters extension. To evaluate the normalized emittance increase due to the pressure bump, the procedure developed in [20] can be used. In the case of a proton beam and injected nitrogen, this gives the formula

$$
\frac{\mathrm{d} \epsilon_{\text {norm-rms }}}{\mathrm{d} s}=0.29 \frac{P}{\beta^{3} \gamma c} \underline{\beta}\left[\frac{\mathrm{mrad}}{\mathrm{m}}\right],
$$

where $P$ is the pressure, $\beta$ and $\gamma$ are the relativistic factors, $c$ the light velocity, and $\beta$ the average Twiss parameter in the pressure bump zone. To give an estimation, let us consider nitrogen injection at $10^{-6}$ Torr for the LHC

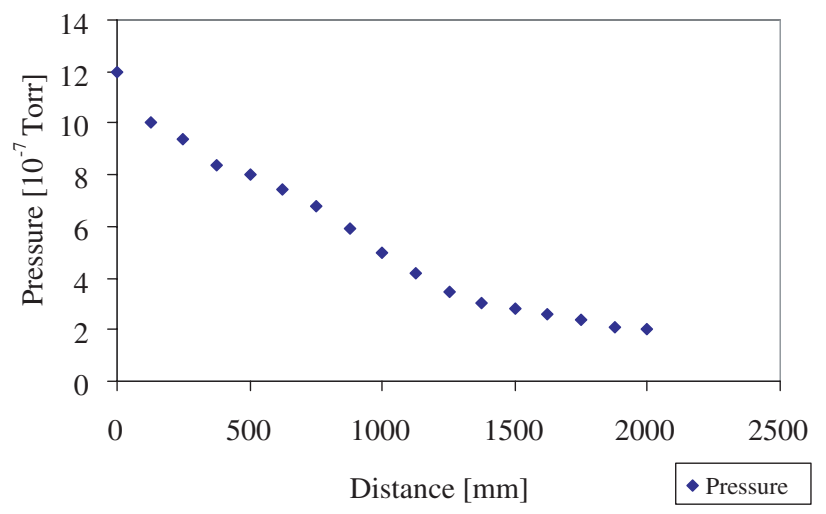

FIG. 17. (Color) Pressure bump vs distance from detector position. beam $(\beta \sim 1$ and $\gamma=7462)$ in a zone in which $\beta=$ $100 \mathrm{~m}$. This will produce a normalized emittance blowup of $5.2 \times 10^{-12} \mu \mathrm{m} /$ turn which means $\sim 6 \times$ $10^{-7} \mu \mathrm{m} / \mathrm{sec}$ which is negligible with respect to the expected emittances.

Another important factor to consider in the LHC is the generation of radiation due to beam-gas interactions. Simulations [21] have shown that a nitrogen pressure bump of the order of $10^{-8}$ Torr, extending for $5.5 \mathrm{~m}$, increases the radiation dose by approximately $3 \%$ which is acceptable.

\section{CONCLUSIONS}

The BPL monitor is a nondestructive diagnostic tool that performs beam profile acquisitions utilizing the luminescent emission produced by the passage of a proton or ion beam in nitrogen gas. It was tested and studied in the framework of the LHC project where nonintercepting diagnostics are required for ultrarelativistic proton and ion beams. The resolution estimation shows that this kind of monitor can be installed in LHC with good imaging results. Total image broadening was estimated at, respectively, $0.04 \%$ (FWHM) or $1.3 \%(4 \sigma)$ for a $\sigma=1 \mathrm{~mm}$ beam size. The BPL principle was tested for the first time with high-energy proton beams in the SPS ring, in an energy range where it was assumed that the emitted photon flux was not sufficient owing to the low cross section. The imaging results were studied and give good results for both proton and lead ion beams. As far as the beam size measurements are concerned, the monitor showed good agreement with the rotative wire scanner $(17 \%)$ with the uncertainty on the beta function in the BPL and WS locations. Better comparisons can be expected after installing a WS next to the BPL. The beam emittance blowup during the SPS acceleration ramp was measured. In this phase the beam size can vary from 5 to $0.8 \mathrm{~mm}(\mathrm{H})$ and from 2.5 to $0.67 \mathrm{~mm}(\mathrm{~V})$. Usually an injected nitrogen bump of $5 \times 10^{-7}$ Torr was used but, to explore the lower pressure limit, a statistical routine was developed allowing a good quality acquisition at $5 \times 10^{-8}$ Torr. The timing resolution permitted measurements for each $\mathrm{GeV}$ in the ramp. From these results it was concluded that a luminescence monitor can provide very good profile measurements for protons and lead ion beams in the LHC with an acceptable influence on the operation of the accelerator.

After assessing that the BPL can be exploited as a nonintercepting monitor in the LHC, some basic experiments on luminescence phenomena were carried out. Cross sections were measured for different energies during the acceleration ramp. To explain the differences with the simple Bethe-Bloch scaling an investigation on angular dependence of the emitted light was carried out. The relative behavior seems to indicate that there is a privileged direction of emission, but this is not sufficient to justify the difference with the low-energy cross-section measure- 
ments. Taking into account the energy extracted by the knock-on electrons brings the difference down, but the uncertainty on the nitrogen pressure is the dominant error. Decay time and afterglow were estimated at, respectively, $55 \mathrm{~ns}$ and 4-8 $\mu \mathrm{s}$. From the decay time measurements an estimation of the bunch length can also be provided. The test with different chromatic filters confirms the spectroscopy lines of emission (391 and $427 \mathrm{~nm}$ ) and the linearity of photon emission versus nitrogen pressure indicates a one-step process. Finally, an estimation of the effect of the pressure bump on beam emittance was provided.

In summary, it can be said that our study has provided a complete characterization of the gas luminescence as nondestructive diagnostic at relativistic energies. The analysis was carried out in an unexplored beam energy domain. For the first time, the obtained beam profile resolution has been evaluated as a function of the beam energy and emittance carefully taking into account the hardware contributions to the resolution function. Estimations for the imaging performances in the LHC beam case have been calculated taking into account the measured probability of emission and the effect of the pressure bump has been estimated. The full description of the hardware setup and of the applied experimental techniques has been provided. Confirmation on cross section and decay time for the nitrogen molecule has been given together with a first estimation for the angular dependence, emission linearity and afterglow effect. Apart from any consideration on the LHC monitoring, this article is an attempt to give a complete review of technical and theoretical considerations to be applied to any luminescence profile monitor.

\section{ACKNOWLEDGMENTS}

We gratefully acknowledge the help of all the members of the SL/BI/PM section and of the LHC/VAC group.
[1] L. G. Christophorou, Atomic and Molecular Radiation Physics (Wiley Interscience, New York, 1971).

[2] E. Jones, R. Kofler, and P. Strolin, CERN Report No. ISRVA/73-57, 1973.

[3] D. D. Chamberlin, G. N. Minerbo, L.E. Teel, and J.D. Gilpatrick, Particle Accelerator Conference Proceeding (PAC), Washington, DC, 1981 [IEEE Trans. Nucl. Sci. 28, 2347 (1981)].

[4] R. H. Huges, J. L. Philpot, and C. Y. Fan, Phys. Rev. 123, 2084 (1961).

[5] L. W. Dotchin, E. L. Chupp, and D. J. Pegg, J. Chem. Phys. 59, 3960 (1973).

[6] J. S. Fraser, IEEE Trans. Nucl. Sci. 28, 2137 (1981).

[7] M. Plum, J. Bosser, E. Bravin, and R. Maccaferri, Nucl. Instrum. Methods Phys. Res., Sect. A 492, 74 (2002).

[8] G. Burtin et al., EPAC Proceedings on the Luminescence Profile Monitor of the CERN SPS, Vienna, 2000.

[9] J.D. Jackson, Classical Electrodynamics (John Wiley \& Sons. Inc., New York, 1999), 3rd ed.

[10] P. Rullhusen, X. Artru, and P. Dhez, Novel Radiation Sources Using Relativistic Electrons, Synchrotron Radiation Techniques and Applications (World Scientific, Singapore, 1998).

[11] A. Variola, Ph.D. thesis, Universite de Paris Sud, 1998.

[12] F. Hornstra, Desy-Hera 89-04, 1989.

[13] A. Variola, A. Arauzo Garcia, C. Bovet, and J. Koopman, in Proceedings La Biodola, Isola d'Elba, Italy, 2000 [special issue of Nucl. Instrum. Methods Phys. Res., Sect. A 461, Nos. 1-3 (2001)].

[14] J. Koopman (private communication).

[15] P.F. Tavares, CERN Report No. CERN-PS/92-55(LP), 1992.

[16] F. Ruggiero, EPAC Proceedings, Stockholm, Sweden, 1998.

[17] Zemax Code by Optima Research.

[18] M. Jimenez (private communication).

[19] D. E. Groom et al., Rev. Part. Phys. 15, 1 (2000).

[20] M. Reiser, The Theory and Design of Charged Particle Beams, Beam Physics and Accelerator Technology (Wiley, New York, 1994).

[21] K. Tsoulou (private communication). 